\title{
ANÁLISE DA VIABILIDADE ECONÔMICA DA PRODUÇÃO DE GOIABA EM CACHOEIRAS DE MACACU - RJ
}

Lidia Satiko Koseki Uesu'1, Daiane Cecchin², Márcio Yoshimitsu Koseki Uesu³, Marcio Kenji Ogino ${ }^{4}$, Carlos Rodrigues Pereira ${ }^{5}$

${ }^{1}$ Mestranda em Engenharia de Biossistemas, Programa de Pós-Graduação em Engenharia de Biossistemas da Universidade Federal Fluminense, Niterói, Brasil, lidia_satiko@id.uff.br

${ }^{2}$ Docente no Departamento de Engenharia Agrícola e do Meio Ambiente da Universidade

Federal Fluminense, Niterói, Brasil

${ }^{3}$ Administrador, Universidade Estácio de Sá, Niterói, Brasil

${ }^{4}$ Engenheiro Agrícola, Universidade Federal Fluminense, Niterói, Brasil

${ }^{5}$ Docente no Departamento de Engenharia Agrícola e do Meio Ambiente da Universidade

Federal Fluminense, Niterói, Brasil

Recebido em: 22/09/2018 - Aprovado em: 23/11/2018 - Publicado em: 03/12/2018

DOI: 10.18677/EnciBio_2018B5

\section{RESUMO}

Dentre os problemas enfrentados no cultivo da goiaba, pode-se destacar, por sua importância, o investimento inicial necessário da atividade, o qual deve ser comparado com outras oportunidades, uma vez que, dependendo da oferta, os preços podem variar e a recuperação do investimento poderá ser demorada, gerando insegurança para o empreendedor. O presente trabalho teve como objetivo analisar a viabilidade econômica da produção de goiaba comercial em Cachoeiras de Macacu, RJ. O estudo de caso foi embasado em dados obtidos de uma propriedade produtora de goiaba de mesa, localizada no município de Cachoeiras de Macacu. O investimento inicial foi de $\mathrm{R} \$ 45.713,00$, sendo considerada a Taxa Mínima de Atratividade (TMA) de 7,01\%, obtendo-se assim, um Valor Presente Líquido (VPL) de $\mathrm{R} \$ 49.167,51$ para um horizonte de 15 anos. Obteve-se uma Taxa Interna de Retorno (TIR) de 12\%, maior que a TMA, demonstrando a viabilidade do investimento.

PALAVRAS-CHAVE: administração rural, fruticultura, investimento.

\section{ANALYSIS OF THE ECONOMIC FEASIBILITY OF GUAVA PRODUCTION IN CACHOEIRAS DE MACACU -RJ}

\begin{abstract}
Among the problems faced in the cultivation of guava, one can highlight the initial investment necessary for the activity, which should be compared in other opportunity, since, depending on the supply, prices may vary and the recovery of the investment may be time consuming, creating insecurity for the entrepreneur. The objective of this study was to analyze the economic viability of commercial guava production in Cachoeiras de Macacu, RJ. The case study was based on data obtained from a guava producing property, located in the municipality of Cachoeiras de Macacu. The initial investment was $\mathrm{R} \$ 45,713.00$, considering the Minimum Acceptable Rate of Return (MARR) of 7,01\%, resulting in a Net Present Value (NPV) of $R \$ 49.167,51$ for a 15-year horizon. An Internal Rate of Return (IRR) of $12 \%$ was obtained, higher than the MARR, demonstrating the investment feasibility.
\end{abstract}

KEYWORDS: investment, rural administration, fruticulture.

ENCICLOPÉDIA BIOSFERA, Centro Científico Conhecer - Goiânia, v.15 n.28; p. 48 


\section{INTRODUÇÃO}

A goiabeira, Psidium guajava L. (Myrtaceae), é cultivada em mais de 50 países das regiões tropicais e subtropicais do mundo. O Brasil possui relevância dentre os maiores produtores mundiais (SOUZA et al., 2016), ficando em primeiro lugar em produção de goiabas vermelhas enquanto a Índia está em primeiro lugar na produção de goiabas brancas (SEBRAE, 2016).

Sua produção é destinada principalmente ao consumo in natura, demanda em grande expansão já que é uma fruta muito saborosa e apreciada pela população brasileira. Além do mais a goiaba é bastante utilizada na fabricação de doces (goiabada, compota ou geleia) (SOUZA et al., 2016). O consumo da fruta é estimado em $300 \mathrm{~g} /$ habitante/ ano (SEBRAE, 2016).

É também uma fonte muito rica em vitamina $C$, carboidrato, ferro e contém uma considerável quantidade de cálcio e fósforo (YADAV et al., 2017). A planta tem sido amplamente estudada em termos de atividade farmacológica de seus principais componentes, e os resultados indicam potente atividade antidiarreica, antihipertensiva, hepatoprotetora, antioxidante, antimicrobiana, hipoglicemiante e antimutagênica (SHRUTHI et al., 2013).

Segundo Arshiya (2013), frutas ricas em antioxidantes ajudam na redução de incidência de doenças degenerativas, como câncer, artrite, arteriosclerose, doença cardíaca, inflamação, disfunção cerebral e o processo de envelhecimento. Em 2016 foram ofertadas 15.586 toneladas da fruta (IBGE, 2017) no Rio de Janeiro, sendo Cachoeiras de Macacu o principal município ofertante, com $54,5 \%$ do total de produção do estado (CEASA - RJ, 2016).

A fruticultura, como no caso da cultura da goiabeira, tem sido uma das atividades agrícolas que mais demandam conhecimentos relativos à irrigação, principalmente devido à utilização de fruteiras de alto valor econômico. A sua utilização é uma estratégia dos fruticultores para reduzir os riscos associados à atividade (MANTOVANI et al., 2003).

Estudos analisando a viabilidade econômica da cultura da goiaba são escassos na literatura mais recente, o que torna este trabalho importante, principalmente para servir como ferramenta de orientação para outros estudos e para investidores nessa área de grande importância do setor agronômico.

Dessa forma, o objetivo do presente trabalho foi estudar a viabilidade econômica da implantação da cultura da goiaba comercial da variedade Ichijo para um período de 15 anos em Cachoeiras de Macacu/ RJ, tendo como principal fonte de escoamento o CEASA- Irajá/RJ.

\section{MATERIAL E MÉTODOS}

Os dados para a elaboração do trabalho foram obtidos por meio de um estudo de caso realizado em uma propriedade comercial de goiabeiras em Cachoeiras de Macacu - RJ, cujas coordenadas geográficas são 22ํ34'42" de latitude sul e $42^{\circ} 43^{\prime} 06$ " de longitude oeste, aproximadamente a $85 \mathrm{~km}$ da capital do Rio de Janeiro.

O clima da região, pela classificação de Köppen, foi identificado como do tipo Af, ou seja, tropical com verão chuvoso e inverno seco, sem uma estação marcadamente seca. A precipitação média mensal varia de $337,8 \mathrm{~mm}$ (fevereiro) a $59,3 \mathrm{~mm}$ (julho), com total anual de $2.050 \mathrm{~mm}$. A temperatura média anual é de 21,9 , sendo janeiro o mês mais quente $\left(25,3^{\circ} \mathrm{C}\right)$, e julho, o mês mais frio $\left(17,9^{\circ} \mathrm{C}\right)$ (AZEVEDO et al., 2018).

Para desenvolver o estudo de viabilidade econômica do plantio da goiaba, foi delimitado um horizonte de 15 anos para o fluxo de caixa, sendo composto pelo 
levantamento de custos conforme a região estudada, como também uma análise a partir dos dados fornecidos pelo proprietário.

Para o cenário referido foi considerado a contratação de 1(um) funcionário fixo com carteira assinada para auxiliar nas atividades de implantação e produção juntamente com o proprietário - responsável também pela parte administrativa da atividade. Para uma produção satisfatória, foram utilizadas a instalação de uma rede de irrigação de microaspersão, e uma casa de bomba com seus respectivos componentes, como a bomba, hidrômetro e nanômetro, conforme exigido pelo INEA (Instituto Estado do Ambiente). Foi considerada a compra de 200 mudas de goiaba da variedade Ichijo com o espaçamento de $7 \times 7 \mathrm{~m}$, havendo 20 linhas com $7 \mathrm{~m}$ de distância entre elas e com $70 \mathrm{~m}$ de comprimento, resultando em aproximadamente 1 ha.

Para a análise de viabilidade econômica do investimento da goiabeira, foi elaborado um fluxo de caixa com os valores dos custos fixos e variáveis com sua respectiva receita gerada. O Valor Presente Líquido (VPL) é o valor dos fluxos de caixa subtraído do valor do investimento inicial. Caso o valor presente das entradas for, no mínimo, igual ao valor presente das saídas, então o investimento é viável. A partir do fluxo de caixa, foi determinada a Taxa Interna de Retorno (TIR), que consiste na taxa de desconto que faz com que o VPL de investimento seja igual a $\$ 0$ já que o valor presente das entradas de caixa iguala-se ao investimento inicial. Os critérios do VPL e da TIR baseiam-se em fluxos de caixa descontados a uma determinada taxa. Esta taxa é denominada de TMA, ou seja, o retorno mínimo exigido para o projeto de investimento (PEREIRA; ALMEIDA, 2008), onde foi admitido $7,01 \%$ ao ano.

\section{RESULTADOS E DISCUSSÃO}

Para uma pessoa que esteja interessada em investir na produção de goiaba comercial, o investimento inicial para 1 hectare de pomar pode chegar a $\mathrm{R} \$ 45.713,00$ (Tabela 1). Deve-se destacar que as maiores despesas são oriundas da aquisição do microtrator e do sistema de irrigação, com $70 \%$ e $18 \%$ respectivamente. Vale salientar que a proporção em relação a irrigação mudará caso seja considerado áreas maiores. O terreno não foi considerado e, caso seja necessário, pode ser realizado por meio de arrendamento.

TABELA 1. Valores de investimento para a implementação de pomar de goiabeira, Cachoeiras de Macacu/ RJ, 2017

\begin{tabular}{|c|c|c|c|}
\hline ITEM & QUANTIDADE & $\begin{array}{c}\text { VALOR } \\
\text { UNITÁRIO } \\
(R \$)\end{array}$ & $\begin{array}{c}\text { VALOR } \\
\text { TOTAL (R\$) }\end{array}$ \\
\hline Sistema de Irrigação & - & (2) & $8.137,20$ \\
\hline Hidrômetro & 1 & 899,10 & 899,10 \\
\hline Manômetro & 1 & 45,00 & 45,00 \\
\hline Bomba $1 \mathrm{cv}$ & 1 & 579,90 & 579,90 \\
\hline Tubulação 0,5" - 50m & 28 & 198,00 & $5.623,20$ \\
\hline $\begin{array}{l}\text { Haste, microaspersor bailarina e micro } \\
\text { tubo }\end{array}$ & 200 & 4,95 & 990.00 \\
\hline $\begin{array}{l}\text { Construção da Casa de Bomba } \\
\text { Muda de goiaba }\end{array}$ & $\begin{array}{c}1 \\
200\end{array}$ & $\begin{array}{c}750,00 \\
20,00\end{array}$ & $\begin{array}{l}750,00 \\
4.000 .00\end{array}$ \\
\hline Equipamentos & - & - & 645,80 \\
\hline Tesoura de Poda & 2 & 81,00 & 162,00 \\
\hline Serrote & 2 & 117,00 & 234,00 \\
\hline ENCICLOPÉDIA BIOSFERA, Centro Científico & nhecer - Goiâ & ר.28: $\mathrm{c}$ & 2018 \\
\hline
\end{tabular}




\begin{tabular}{lccc} 
Garrafa Térmica & 2 & 18,70 & 37,40 \\
Enxada & 2 & 41,40 & 82,80 \\
Facão & 2 & 64,80 & 129,60 \\
Caixa Plástica & $\mathbf{1 0}$ & 18,00 & $\mathbf{1 8 0 , 0 0}$ \\
Microtrator 14 cv & $\mathbf{1}$ & $32.000,00$ & $\mathbf{3 2 . 0 0 0 , 0 0}$ \\
\hline \multicolumn{1}{c}{ CUSTO TOTAL } & & & $\mathbf{4 5 . 7 1 3 , 0 0}$ \\
\hline
\end{tabular}

Fonte: Elaborado pelos autores

Um funcionário da área rural ganha em média $R \$ 1.371,60$, no qual irá auxiliar no cultivar da goiabeira. O produtor teve um pró-labore com remuneração mensal de $\mathrm{R} \$ 1.200,00$ e um gasto de $\mathrm{R} \$ 35,00$ referente ao ITR (Imposto sobre a Propriedade Territorial Rural). Foi observado o consumo de $100 \mathrm{KW} / \mathrm{mês}$ de energia para 1 ha, considerando que a bomba de irrigação esteja ligada a uma rede de energia elétrica. Estes valores são referentes ao custo fixo do processo (Tabela 2).

TABELA 2. Custo fixo para a implementação de pomar de goiabeira, Cachoeiras de Macacu/ RJ, 2017

\begin{tabular}{|c|c|c|c|c|c|c|c|c|c|}
\hline \multirow[t]{2}{*}{ ITEM } & \multicolumn{3}{|c|}{ ANO 0} & \multicolumn{2}{|c|}{$\begin{array}{c}\text { ANO DE } \\
\text { IMPLANTAÇÃO }\end{array}$} & \multicolumn{2}{|c|}{ ANO 2} & \multicolumn{2}{|c|}{ ANO 3} \\
\hline & Quant. & $\begin{array}{l}\text { Valor } \\
\text { Unit. } \\
(\mathrm{R} \$)\end{array}$ & $\begin{array}{l}\text { Valor } \\
\text { Total } \\
(\mathrm{R} \$) \\
\end{array}$ & $\begin{array}{l}\text { Valor } \\
\text { Unit. } \\
(\mathrm{R} \$) \\
\end{array}$ & $\begin{array}{l}\text { Valor } \\
\text { Total } \\
(\mathrm{R} \$)\end{array}$ & $\begin{array}{l}\text { Valor } \\
\text { Unit. } \\
(\mathrm{R} \$)\end{array}$ & $\begin{array}{l}\text { Valor } \\
\text { Total } \\
(\mathrm{R} \$) \\
\end{array}$ & $\begin{array}{l}\text { Valor } \\
\text { Unit. } \\
(\mathrm{R} \$)\end{array}$ & $\begin{array}{l}\text { Valor } \\
\text { Total } \\
(\mathrm{R} \$) \\
\end{array}$ \\
\hline $\begin{array}{l}\text { Salário do } \\
\text { Funcionário }\end{array}$ & 13 & & & $1.371,60$ & $17.830,80$ & $1.371,60$ & $17.830,80$ & $1.371,60$ & $17.830,80$ \\
\hline Pró-Labore & 12 & & & $1.200,00$ & $14.400,00$ & $1.200,00$ & $14.400,00$ & $1.200,00$ & $14.400,00$ \\
\hline ITR & 1 & & & 35,00 & 35,00 & 35,00 & 35,00 & 35,00 & 35,00 \\
\hline $\begin{array}{l}\text { Depreciação } \\
\text { Energia }\end{array}$ & 246 & & & 14,93 & $3.679,85$ & 14,93 & $3.679,85$ & 14,93 & $3.679,85$ \\
\hline $\begin{array}{l}\text { Elétrica } \\
\text { VALOR } \\
\text { TOTAL }\end{array}$ & 12 & & & 59,00 & $\begin{array}{c}708,00 \\
36.653,65\end{array}$ & 59,00 & $\begin{array}{c}708,00 \\
36.653,65\end{array}$ & 59,00 & 708,00 \\
\hline
\end{tabular}

Fonte: Elaborado pelos autores

A produção alcançada no ano de 2017 com a variedade Ichijo foi de 60 ton.ha ${ }^{-1}$. Este resultado está acima do que Neto (2007) verificou no Nordeste com a variedade Paluma - 40 ton à 50 ton.ha ${ }^{-1}$.ano ${ }^{-1}$-, considerando as mesmas condições de manejo, como a irrigação e poda de frutificação. Segundo Galdino et al. (2016), a classificação de Köppen para Petrolina é Ams. Assim, o clima é caracterizado pela escassez e irregularidade das precipitações com chuvas no verão e forte evaporação, como consequência das altas temperaturas. Enquanto Cachoeiras de Macacu apresenta temperatura média do ar $\left(21,9^{\circ} \mathrm{C}\right)$ e alta pluviosidade (AZEVEDO et al., 2018).

Cerca de $18 \%$ da produção total cultivada foi vendida diretamente a uma rede de varejo com o preço médio de $R \$ 3,10 / \mathrm{kg}$. A goiaba seguiu a mesma padronização de tamanho, coloração esverdeada e sem qualquer tipo de dano em sua superfície, seja proveniente de doenças ou danos físicos causados durante a embalagem ou até mesmo em seu transporte à packing house. Pode-se verificar na figura 10 sistema mencionado, onde não há a influência do intermediário na venda da mercadoria. 


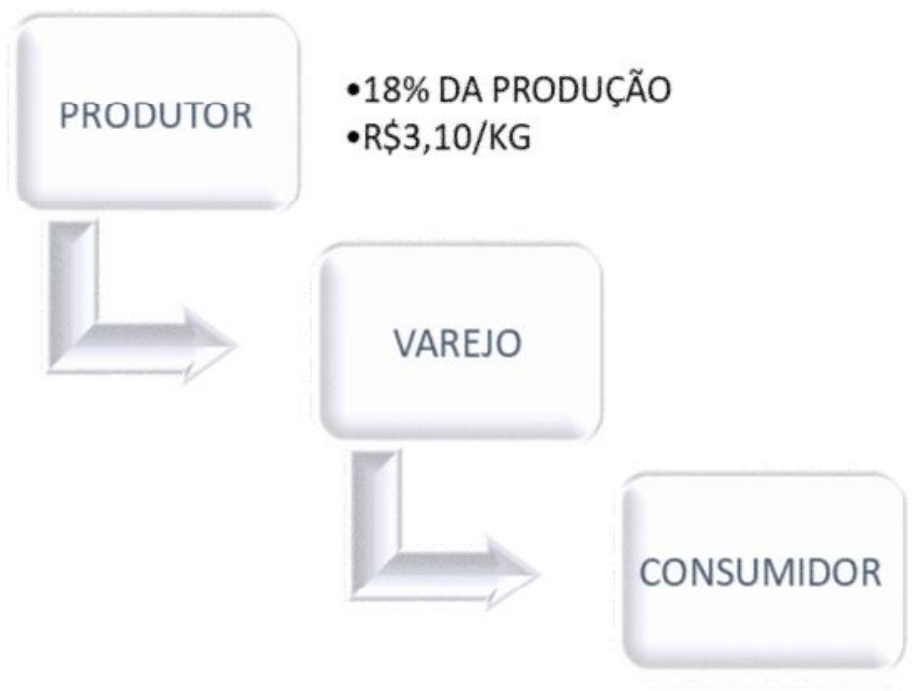

FIGURA 1: Esquema do processo de venda do produto direto para o varejo (Produtor-Loja)

Já os outros $82 \%$ da produção foram comercializadas no CEASA - Irajá/RJ com um preço médio de venda de $R \$ 1,33 / \mathrm{kg}$, valor inferior, ao obtido no comércio de goiabas no CEAGESP, de $\mathrm{R} \$ 1,85 / \mathrm{kg}$ (TOKAIRIN, 2014). O conjunto de caixas de goiabas comercializado no CEASA, é composto por goiabas do mesmo padrão daquelas comercializadas no sistema de varejo (Produtor-Loja) goiabas de menor tamanho, goiabas maduras e por aquelas que possuem algum tipo de defeito em sua superfície, mas que não compromete o seu consumo, cada classificação separada em caixas distintas. Na figura 2, é apresentado o sistema de envio do produto para o CEASA, em que o produtor repassa uma comissão de $10 \%$ sobre o valor médio do preço/quilograma ao atravessador, conforme as vendas efetuadas de seu produto para um varejista, incidindo sobre o preço final ofertado ao consumidor. No entanto, de acordo com o CEASA (2017), a precificação média do quilograma da goiaba foi de $R \$ 5,26$, havendo grande disparidade entre os valores recebidos pelo produtor e o valor disponível para consulta. Essa diferença, de $74,71 \%$, valor perdido pelo produtor, ainda é aumentada pelos $10 \%$ que são pagos ao atravessador (representante de vendas), passando para $77,24 \%$ por quilograma de goiaba, valor publicado em relação ao valor real recebido pelo produtor. Esse valor, $\mathrm{R} \$ 4,06$, é considerado perdido comparado à situação teórica da venda ser efetuada para o lojista, pelo valor publicado pelo CEASA, ou seja, de $\mathrm{R} \$ 5,26$. 


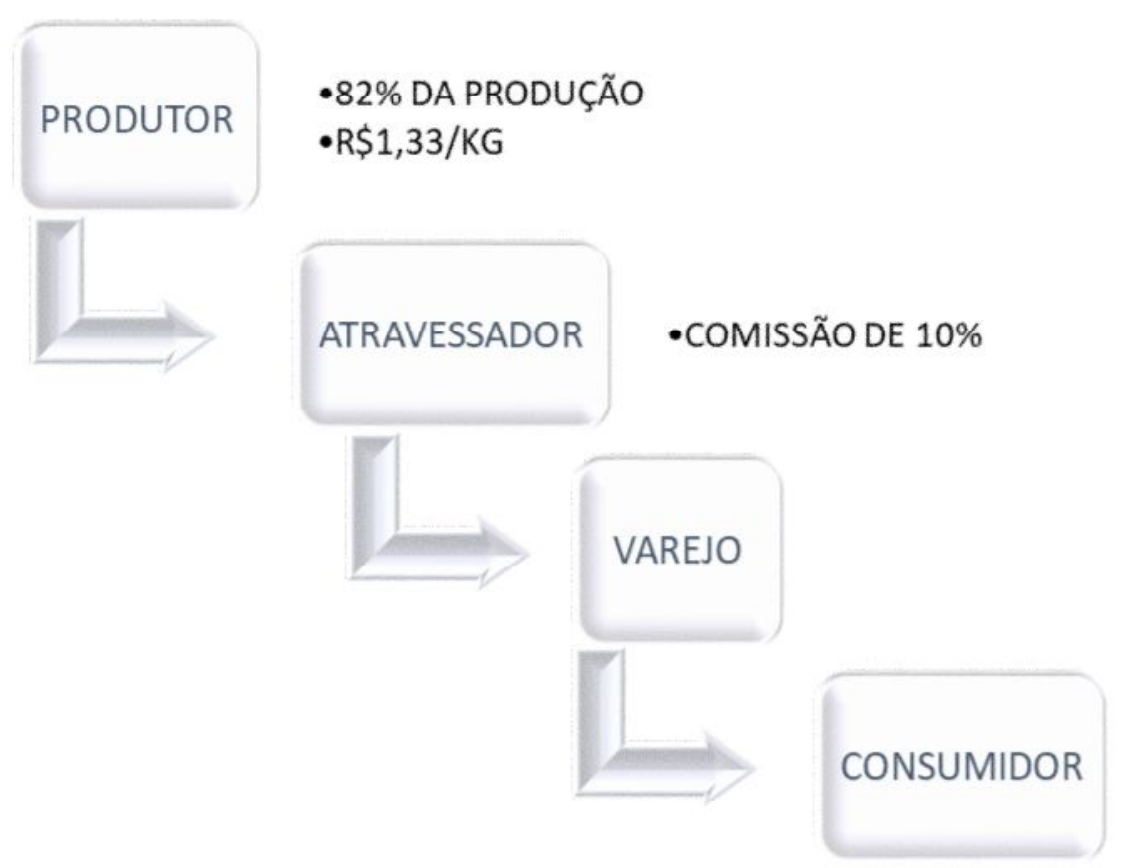

FIGURA 2: Esquema do processo de venda do produto utilizando o CEASA (Produtor-CEASA-Loja).

Portanto, se o produtor conseguisse mais autonomia em sua venda, o lucro poderia aumentar em até $233 \%$ (comparação entre o sistema Produtor-Loja $(R \$ 3,10)$ com o sistema Produtor-CEASA-Loja $(\mathrm{R} \$ 1,33))$. Assim, é importante analisar a forma de comercialização de seus produtos buscando evitar a dependência de intermediários para a realização deste trabalho.

Segundo recomendação agronômica, para o controle fitossanitário foi considerada a utilização de $250 \mathrm{ml}$ de inseticida diluído em 1.000 litros de água. ha ${ }^{-1}$. Logo aplica-se 5litros/planta quando há qualquer infestação. Dessa forma sua aplicação foi realizada cerca de quatro vezes por ano, podendo assim, o volume de um litro durar até um ano. Foi considerado o mesmo processo para o fungicida, com a concentração de 0,6 litros do produto em 1.000 litros de água. $O$ valor de cada produto foi de $R \$ 211,50$ e $R \$ 114,30$ respectivamente.

Segundo Bertani et al. (2011), a partir do terceiro ano de idade, pode-se considerar a fase de produção plena da goiabeira, sendo adotado esta consideração no fluxo de caixa. A produção, comercializada no CEASA Irajá/RJ, teve como um dos principais gastos, a comissão cobrada pelo representante de vendas, correspondente a $10 \%$ do valor de venda do produto. E, conforme a afirmação de Tokairin (2014), outro item de custo considerável foi o frete, com despesa de $\mathrm{R} \$ 3,50$ por caixa ou 19,4 centavos/kg (Tabela 3).

Para trabalhos que necessitam de maior potência do trator, como a utilização do pulverizador, roçadeira e triturador, foi considerado a locação do mesmo, com potência de $75 \mathrm{cv}$, tração 4 × 4 a um valor de $R \$ 120,00 /$ hora (preço praticado na região). Para estes trabalhos, foi considerado $23 \mathrm{~h}$ trabalhado em um ano/hectare, sendo 6 horas/ano para a pulverização, 7,5 horas/ano de roçadeira e 9,5 horas/ ano de triturador. 
TABELA 3. Custo variável para a implementação de pomar de goiabeira, Cachoeiras de Macacu/ RJ, 2017

\begin{tabular}{|c|c|c|c|c|c|c|c|c|c|c|c|}
\hline \multirow[t]{2}{*}{ ITEM } & \multicolumn{3}{|c|}{ ANO 0} & \multicolumn{2}{|c|}{$\begin{array}{c}\text { ANO DE } \\
\text { IMPLANTAÇÃO }\end{array}$} & \multicolumn{2}{|c|}{ ANO 2} & \multicolumn{2}{|c|}{ ANO 3} & \multicolumn{2}{|c|}{ ANO 4} \\
\hline & Quant. & $\begin{array}{l}\text { Valor } \\
\text { Unit. } \\
(\mathrm{R} \$)\end{array}$ & $\begin{array}{l}\text { Valor } \\
\text { Total } \\
(\mathrm{R} \$)\end{array}$ & $\begin{array}{l}\text { Valor } \\
\text { Unit. } \\
(\mathrm{R} \$)\end{array}$ & $\begin{array}{l}\text { Valor } \\
\text { Total } \\
(\mathrm{R} \$)\end{array}$ & $\begin{array}{l}\text { Valor } \\
\text { Unit. } \\
(\mathrm{R} \$)\end{array}$ & $\begin{array}{l}\text { Valor } \\
\text { Total } \\
(\mathrm{R} \$)\end{array}$ & $\begin{array}{l}\text { Valor } \\
\text { Unit. } \\
(\mathrm{R} \$)\end{array}$ & $\begin{array}{l}\text { Valor } \\
\text { Total } \\
(\mathrm{R} \$)\end{array}$ & $\begin{array}{l}\text { Valor } \\
\text { Unit. } \\
(\mathrm{R} \$)\end{array}$ & $\begin{array}{l}\text { Valor } \\
\text { Total } \\
(\mathrm{R} \$)\end{array}$ \\
\hline $\begin{array}{l}\text { Embalagem } \\
\text { Comissão do }\end{array}$ & 3335 & & & 0,00 & 0,00 & 0,00 & 0,00 & 3,37 & $11.238,81$ & 3,37 & $11.238,81$ \\
\hline vendedor & $10 \%$ & & & 0,00 & 0,00 & 0,00 & 0,00 & & $6.564,00$ & & $6.564,00$ \\
\hline $\begin{array}{l}\text { Frete } \\
\text { Preparo de solo }\end{array}$ & 3335 & & & 0,00 & 0,00 & 0,00 & 0,00 & 3,50 & $11.672,35$ & 3,50 & $11.672,35$ \\
\hline $\begin{array}{l}\text { e plantio } \\
\text { Insumos }\end{array}$ & 1 & & & $1.000,00$ & $1.000,00$ & 0,00 & 0,00 & 0,00 & 0,00 & 0,00 & 0,00 \\
\hline Agrícolas & 4 & & & 537,30 & $2.149,20$ & 537,30 & $2.149,20$ & 537,30 & $2.149,20$ & 537,30 & $2.149,20$ \\
\hline $\begin{array}{l}\text { Combustível } \\
\text { Manutenção }\end{array}$ & 150 & & & 3,63 & 544,50 & 3,63 & 544,50 & 3,63 & 544,50 & 3,63 & 544,50 \\
\hline dos equip. & 2880 & & & 0,45 & $1.296,00$ & 0,45 & $1.296,00$ & 0,45 & $1.296,00$ & 0,45 & $1.296,00$ \\
\hline $\begin{array}{l}\text { Tratos Culturais } \\
\text { Aluguel do }\end{array}$ & 2 & & & 226,67 & 453,33 & 226,67 & 453,33 & 226,67 & 453,33 & 226,67 & 453,33 \\
\hline trator $(39 \mathrm{cv})$ & $23 \mathrm{~h}$ & & & 120,00 & $2.720,00$ & 120,00 & $2.720,00$ & 120,00 & $2.720,00$ & 120,00 & $2.720,00$ \\
\hline $\begin{array}{l}\text { Fitossanitário } \\
\text { Análises (Água }\end{array}$ & 1 & & & 350,80 & 350,80 & 350,80 & 350,80 & 350,80 & 350,80 & 350,80 & 350,80 \\
\hline e solo) & 1 & & & 76,00 & 76,00 & 76,00 & 76,00 & 76,00 & 76,00 & 76,00 & 76,00 \\
\hline EPI & 2 & & & 262,37 & 524,74 & 262,37 & 524,74 & 262,37 & 524,74 & 262,37 & 524,74 \\
\hline TOTAL & & & & & $9.114,57$ & & $8.114,57$ & & $37.589,73$ & & $37.589,73$ \\
\hline
\end{tabular}

Fonte: Elaborado pelos autores 
Observa-se no fluxo de caixa (Tabela 4) que, a partir do terceiro ano, recuperamse os investimentos iniciais, visto que coincide com o ano de início da produção plena.

TABELA 4. Fluxo de caixa para um pomar de goiabeira durante 15 anos

\begin{tabular}{cc}
\hline \multicolumn{2}{c}{ FLUXO DE CAIXA } \\
\hline ANO & $\mathrm{R} \$ . \mathrm{ha}^{-1}$ \\
\hline 0 & $-45.713,00$ \\
Implantação & $-45.768,22$ \\
2 & $-44.768,22$ \\
3 & $24.876,62$ \\
4 & $24.876,62$ \\
5 & $24.876,62$ \\
6 & $24.876,62$ \\
7 & $24.876,62$ \\
8 & $23.209,14$ \\
9 & $23.209,14$ \\
10 & $23.209,14$ \\
11 & $23.209,14$ \\
12 & $23.209,14$ \\
13 & $23.209,14$ \\
14 & $23.209,14$ \\
15 & $27.297,86$ \\
\hline
\end{tabular}

Fonte: Elaborado pelos autores

Com a taxa mínima de atratividade de 7,01\%, foi obtido o VPL correspondente a $\mathrm{R} \$ 49.167,51$, indicando, assim, que $\mathrm{o}$ investimento inicial foi recuperado. Comparado com a produção de banana, considerado também um produto de grande importância para o município de Cachoeiras de Macacu (CAVALIN; MONTEIRO, 2012) foi adquirido a TMA corresponde a $12 \%$ e o VPL de $R \$ 13.752,80$ (PONCIANO et al., 2004), podendo-se então concluir que a implantação de goiaba é mais viável em virtude dos dados apresentados.

Segundo Pereira e Almeida (2008), se a TIR for maior que o custo de capital (Taxa Mínima de Atratividade), o projeto pode ser aceito. Desse modo, a TIR (12\%) sobrepujou a TMA considerada neste estudo, sugerindo que o projeto pode ser implementado.

\section{CONCLUSÕES}

A análise do fluxo de caixa permitiu o conhecimento do investimento, possibilitando que o produtor possa promover melhorias na gestão financeira, otimizando os investimentos, maximizando assim os lucros da atividade ao longo do tempo.

O investidor deve atentar-se aos diferentes mercados e buscar aquele de maior retorno.

$\mathrm{Na}$ análise realizada, o sistema composto por Produtor-Loja (18\%) e ProdutorCEASA-Loja (82\%), se mostrou viável para a implementação da cultura da goiabeira. 


\section{REFERÊNCIAS}

ARSHIYA, S. The Antioxidant Effect of Certain Fruits: - A Review. Journal of Pharmaceutical Sciences and Research. Chennai, v. 5, n. 12, p. 265-168, 2013. Disponível em: https://pdfs.semanticscholar.org/44f5/93b35b1b24cf92d957a83ba76c36e841a3b2.pd $f>$.

AZEVEDO, A. D.; FRANCELINO, M. R.; CAMARA, R.; PEREIRA, M. G.; LELES, P. S. S. Estoque de carbono em áreas de restauração florestal da mata atlântica. Revista FLORESTA. Curitiba, v. 48, n.2, p. 183-194, 2018. Disponível em: < https://revistas.ufpr.br/floresta/article/view/54447/35165>. Doi: 10.5380/rf.v48 i2.54447.

BERTANI, R. M. A.; FERNANDES, D. M.; BÜLL, L. T. Adubação e calagem. In: SAMPAIO, A. C. (Org.). Goiaba: do plantio à comercialização. 1 ed. Campinas: Editora CATI, 2011. p. 23 - 31. Disponível em: $<\mathrm{ftp}: / /$ ttp.feis.unesp.br/agr/pdf/ivro_goiaba_sampaio_hernandez.pdf>.

CAVALIN, M. O.; MONTEIRO, J. M. G. Boas práticas agrícolas recomendadas para o cultivo de banana na comunidade do Faraó em Cachoeiras de Macacu, RJ. Boletim de Pesquisa e Desenvolvimento (Embrapa Solos), Rio de Janeiro, v. 210, p. 1-37, 2012. Disponível em: < https://ainfo.cnptia.embrapa.br/digital/bitstream/item/103908/1/BPD-210-BoasPraticas-Banana.pdf $>$.

CEASA - RJ - Centrais de Abastecimento do Estado do Rio de Janeiro. Informativo Anual de 2015, Unidade Grande Rio. 2016. Disponível em: <http://www.ceasa.rj.gov.br/ceasa_portal/view/InformativoAnual2015.pdf >.

CEASA - RJ - Centrais de Abastecimento do Estado do Rio de Janeiro. Consulta de Cotação, Unidade Grande Rio. 2017. Disponível em: <http://www.ceasa.rj.gov.br/ceasa_portal/view/ListarCotacoes.asp >.

GALDINO, J. J. B.; ALBUQUERQUE P. H. C. C.; SALAZAR, G. A.; VILELA, O. C.; FRAIDENRAICH, N. Estudo da radiação solar da cidade de petrolina através de um modelo empírico de céu claro. Revista Brasileira de Energia Solar, v.7, n.2, p. 98105, 2016.2 Disponível em:

https://rbens.emnuvens.com.br/rbens/article/view/152/150>.

IBGE - Instituto Brasileiro de Geografia e Estatística. Produção Agrícola Municipal. 2017. Disponível em: < https://sidra.ibge.gov.br/tabela/1613>.

MANTOVANI, E. C.; ZINATO, C. E.; SIMÃO, F. R. Manejo de irrigação e fertirrigação na cultura da goiabeira. In: ROZANE, D. E.; COUTO, F. A. D'A. (Org.). Cultura da goiabeira: tecnologia e mercado. 1ed. Viçosa: Editora UFV, 2003. p. 243-302. Disponível

em:<http://www.nutricaodeplantas.agr.br/site/ensino/pos/Palestras_William/Livrogoia ba_pdf/8_irrigacao.pdf>. 
GONZAGA NETO, L. Produção de goiaba. Fortaleza: Instituto Frutal. 2007. 64 p. Disponível em: <http://files.prof-vanderufersa.webnode.com.br/2000000430232a032cb/produ\%C3\%A7\%C3\%A30\%20de\%20goiaba.pdf>.

PEREIRA, W. A.; ALMEIDA, L. S. Método manual para cálculo da taxa interna de retorno. Rio Verde, Revista Objetiva, v. 4, p. 38-50, 2008. Disponível em: < http://home.ufam.edu.br/andersonlfc/Macrol/MetodoManualTIR.pdf >.

PONCIANO, N. J.; SOUZA, P. M.; MATA, H. T. C.; VIEIRA, J. R.; MORGADO, I. F. Análise de Viabilidade Econômica e de Risco da Fruticultura na Região Norte Fluminense. Revista de Economia e Sociologia Rural, Brasília, v. 42, n.4, p. 615635, 2004. Disponível em: < http://dx.doi.org/10.1590/S0103-20032004000400005>. Doi: 10.1590/S0103-20032004000400005.

SEBRAE - Serviço Brasileiro de Apoio às Micro e Pequenas Empresas. O cultivo e o mercado da goiaba. 2016. Disponível em: <http://www.sebrae.com.br> $<$ http://www.sebrae.com.br/sites/PortalSebrae/artigos/O-cultivo-e-omercado-dagoiaba>.

SHRUTHI, S. D., ROSHAN, A., TIMILSINA, S. S., SUNITA, S. A review on the medicinal plant psidium guajavalinn. (myrtaceae). Journal of Drug Delivery \& Therapeutics. Karnataka, v. 3, n. 2, p. 162-168, 2013. Disponível em: < http://jddtonline.info/index.php/jddt/article/view/404/265 10.22270/jddt.v3i2.404.

SOUZA, J. C.; SILVA, R. A.; SANTA-CECÍLIA, L. V. C.; MATOS, C. S. M. Pragas da goiabeira. Informe Agropecuário. Manejo de pragas de fruteiras tropicais. Belo Horizonte, v. 37, n.293, p. 63, 2016.

TOKAIRIN, T. O.; CAPPELLO, F. P.; SPÓSITO, M. B. Custo de produção de goiabas para mesa produzidas com e sem ensacamento: Estudo de caso. Revista Brasileira de Fruticultura, Jaboticabal, v.36, n.3, p.542-549, 2014. Disponível em: < http://dx.doi.org/10.1590/0100-2945-347/13.> Doi: 10.1590/0100-2945-347/13.

YADAV, K. BARHOLIA, A. KHAN, R. PATEL, R. Effect of foliar spray of borax on growth and yield of guava (Psidium guajava L.) cv. Gwalior-27. International Journal of Chemical Studies. Nova Deli, v. 5, n. 6, p. 495-497, 2017. Disponível em :<http://www.chemijournal.com/archives/2017/vol5issue6/PartG/5-5-315-408.pdf >. 